\title{
Data transmission deployment and reliability analysis in enterprise informatization construction
}

\author{
LI Jun ${ }^{1}$,LIU Xiao-Ling ${ }^{2}$ \\ \{LIjun3812@163.com ${ }^{1}$,LiuXiaoLing3812@163.com $\left.{ }^{2}\right\}$ \\ (1. Institute of Education and Physical Education,Jiangxi Institute of Applied Science and Technology, \\ Nanchang 330100,China; \\ 2.Institute of Education and Physical Education,Jiangxi Institute of Applied Science and Technology, \\ Nanchang 330100,China)
}

\begin{abstract}
In the construction of enterprise informatization, the traditional reliability analysis method of data transmission deployment has the problem of high data packet loss rate, which affects the reliability analysis results.Therefore, data transmission deployment and reliability analysis in enterprise informatization construction are proposed. Firstly, the wireless transmission module is designed to ensure the safe and barrier free transmission of data, and the can interface is designed to connect all links in the information construction with the wireless transmission module. On this basis, data is divided and deployed by data deployment algorithm; path loss of data transmission channel is taken as the basis of reliability analysis, and reliability analysis is completed by calculating various losses. The experimental results show that: compared with the traditional method, the data transmission deployment and reliability analysis method designed in the enterprise information construction has no packet loss, and the data is safe and reliable. This method is suitable for the application in the enterprise information construction.
\end{abstract}

Key words: Information technology; Data transmission; Reliability analysis; Data transmission deployment;

\section{Introduction}

Enterprise is the representative of a country's economic strength, which plays an important role in the process of national economic construction and development. The effective development of enterprises is an important guarantee for the country to realize modernization and strengthen the country[1]. With the development of information technology, enterprise information construction is a common way to improve enterprise efficiency in the 21 st century. Therefore, how to do well in large-scale enterprise information construction has become one of the focuses of scholars in recent years[2].

Enterprise informatization construction refers to the establishment of enterprise informatization planning blueprint based on the planning objectives of the enterprise, combined with the practice of enterprise informatization and the mastery of the development 
trend of information technology, so as to guide the enterprise informatization process comprehensively and systematically. Coordinate the application of information technology, make full and effective use of information resources of enterprises, and meet the needs of business development of enterprises[3]. It is a gradual derivation and definition process from the goal and business process of enterprise information construction to the framework of information system and the implementation strategy of information system. It takes the realization of enterprise information construction goal as the ultimate goal of information and system implementation[4].

In the past, there was data packet loss in the data transmission deployment of enterprise informatization construction, which led to the lack of strong basis for reliability analysis. Therefore, the data transmission deployment and reliability analysis in the enterprise information construction are proposed.

Because the path loss in data transmission is an important index to measure the reliability of enterprise information construction, the evaluation of path loss in data transmission has been widely concerned by researchers. Most of the research on path loss evaluation in data transmission is based on the premise that data is transmitted through disjoint paths. However, in real life, data will inevitably be transmitted through paths containing the same link. Therefore, the reliability of path loss in data transmission is analyzed to judge whether enterprise information construction is reliable.

\section{Data transmission deployment in enterprise information construction}

\subsection{Data transmission deployment in enterprise information construction}

In the enterprise information construction, due to the bad working environment and strong interference, the data transmission module with strong anti-interference ability and error detection and correction ability is selected to ensure the accuracy of data transmission. After experimental comparison and test, str-35 industrial low power wireless data transmission module meets the requirements. The channel center frequency of str-35 wireless data transmission module is $900 \mathrm{MHz}$, which has high anti-interference ability and low bit error rate. The modulation mode based on GFSK and efficient forward error correction channel coding technology are adopted to improve the ability of data anti burst interference and random interference[5]. The module can automatically filter out the false data generated in the air (received is sent). The interface baud rate is 19200bps. Flexible programming, no need to prepare redundant programs, as long as the data from the interface can be sent and received. The technical specifications of STR-35 transmission module are shown in Table 1 below: 
Table 1 technical indicators of transmission module

\begin{tabular}{|c|c|c|c|}
\hline $\begin{array}{l}\text { Numb } \\
\text { er }\end{array}$ & Technical index & parameter & Remark \\
\hline 1 & Modulation & MSK & \\
\hline 2 & working frequency & $\begin{array}{l}300-365 \mathrm{MHz}, \quad 400-480 \mathrm{MHz}, \\
826-968 \mathrm{MHz}\end{array}$ & User set \\
\hline 3 & Transmit power & $0 \mathrm{dbm}, 5 \mathrm{dbm}, 10 \mathrm{dbm}$ & User set \\
\hline 4 & Receiving sensitivity & $-118 \mathrm{dBm}$ & \\
\hline 5 & Emission current & $\leq 27 \mathrm{~mA}$ & \\
\hline 6 & Receive current & $\leq 18 \mathrm{~mA}$ & \\
\hline 7 & Wireless wake-up current & $\leq 8 \mathrm{~mA}$ & \\
\hline 8 & stand-by current & $\leq 20 \mu \mathrm{A}$ & \\
\hline 9 & Interface speed & $1200-115200 \mathrm{bps}$ & \\
\hline 10 & Interface Type & UART & $\begin{array}{l}\text { User } \\
\text { selectable }\end{array}$ \\
\hline 11 & Operating Voltage & $+4.5 \sim 5.5 \mathrm{~V}$ & User set \\
\hline 12 & Operating temperature & $-40^{\circ} \mathrm{C} \sim 85^{\circ} \mathrm{C}$ & \\
\hline 13 & Storage temperature & $-65^{\circ} \mathrm{C} \sim 150^{\circ} \mathrm{C}$ & \\
\hline 14 & Working humidity & $10 \% \sim 90 \%$ Relative humidity & \\
\hline 15 & Number of switches & $\begin{array}{l}4 \text { channels, expandable to } 16 \\
\text { channels }\end{array}$ & \\
\hline 16 & $\begin{array}{l}\text { Maximum controllable } \\
\text { switching voltage }\end{array}$ & $\mathrm{AC} 220 \mathrm{~V}, \mathrm{DC} 30 \mathrm{~V}$ & \\
\hline 17 & $\begin{array}{l}\text { Maximum controllable } \\
\text { switching current }\end{array}$ & $\mathrm{AC} 0.5 \mathrm{~A}, \mathrm{DC} 2 \mathrm{~A}$ & \\
\hline
\end{tabular}

Based on the designed wireless transmission module, the CAN bus interface is designed to connect all links in the enterprise information construction through the CAN bus interface, so as to realize the data transmission between each link.

\subsection{CAN interface design}

The internal link of enterprise information construction is connected by CAN bus, and P82C250 chip is used as CAN bus transceiver in the design[6]. P82C250 is the interface between the CAN protocol controller and the physical bus. The device provides differential transmission capability to the bus and differential reception capability to the CAN controller.

P82C250 has 8 pins, dip package, and the function definition of each pin is shown in Table 2. The eighth pin rs of p82c250 can be grounded or connected to high level through 
different connection modes, so that p82c250 can work in one of three modes of high speed, standby and slope control [7].

Table 2 Pin function definition of P82C250

\begin{tabular}{llll}
\hline Pin & name & Pin & name \\
1 & TXD & 5 & VREF \\
2 & GND & 6 & CANL \\
3 & VCC & 7 & CANH \\
4 & RXD & 8 & RS \\
\hline
\end{tabular}

P87C591 is equipped with CAN controller and CAN interface circuit is designed. The interface between P82C250 and CAN bus adopts certain safety and anti-interference measures. The canh and canl pins of $82 \mathrm{C} 250$ are respectively connected with CAN bus through a $5 \Omega$ resistance, which CAN play a role of current limiting and protect $82 \mathrm{C} 250$ from over-current impact. Two 30pF small capacitors are connected in parallel between CANH and CANL and the ground, which can filter the high-frequency interference on the bus and have certain anti electromagnetic radiation ability[8]. The Rs pin of $82 \mathrm{C} 250$ is connected with a slope resistance, which is adjusted appropriately according to the bus communication speed.

\subsection{Data deployment placement algorithm}

In order to reduce the data transmission overhead across the data center after the data deployment, the data is divided according to the strength of the association dependency between the data, so that the data with strong association relationship can be divided into the same data subset and deployed to the same data center [9]. Due to the large scale of data in enterprise information construction and the limited storage space of data center, it is necessary to consider whether the data subset scale exceeds the maximum storage capacity of a single data center when dividing data [10]. In addition, for the data with fixed deployment location restrictions, since the data center to be deployed has been determined, the deployment location of the data subset composed of the data closely associated with it can also be determined, and the size of the subset can also be determined by the storage space of the data center.

Based on the above analysis, the data center storage space is sorted in descending order. Assuming that the sorted data center sequence is expressed as $\left\{u_{1}, u_{2}, \ldots, u_{\mathrm{n}}\right\}$, the storage space in the data center is sorted as $Q\left(u_{1}\right) \geqslant Q\left(u_{2}\right) \geqslant \ldots \geqslant Q\left(u_{\mathrm{n}}\right)$.

On the basis of the sorting results, the flow of data partition algorithm with limited scale is as follows. For data $r_{\varepsilon} \in R_{\mathcal{E}}$ with fixed deployment restrictions, the size of data subset deployed to data center $\operatorname{cen}\left(r_{\varepsilon}\right)$ is determined according to its storage capacity of deployed data center $\operatorname{cen}\left(r_{\varepsilon}\right)$; According to the relationship between data and $r_{\varepsilon}$ and the size of data, select the members of the data subset from the data set that needs to be deployed to the same 
location and the data set that does not have the limitation of deployment location. Repeat the above process until all data in the $R_{\mathcal{E}}$ set is divided. Secondly, for the data $r_{\tau} \in R_{\tau}$ without the limitation of deployment location, the size of data subset deployed to a single data center is determined according to the maximum storage capacity of the current data center; According to the relationship between data and $r_{\tau}$ and the size of data, the members of the data subset are selected from the data set $R_{\tau}$. Repeat the above process until all data in the $R_{\tau}$ set is divided. At this point, all data is divided into corresponding data subsets.

Repeat the above process until all data members are determined, then the data members that data subset $q_{1}$ should contain are obtained. Then continue to divide and exchange the data of the remaining data members in the original data, which can complete the division of other limited size data subset members, and also ensure the small amount of data transmission between data subsets.

After the data partition, deploy the data subset to the data center. In data deployment, in order to ensure that the data access delay does not exceed the limit, the candidate data center sets available for deployment for each data set are different. Let $F=\left\{r_{1}, r_{2}, \ldots, r_{\mathrm{n}}\right\}$ be the set of all data. Each data in $F$ is represented by a tuple, where $r_{\tau}=\langle$ size,cen $\rangle$ represents the size of the data, size $\left(r_{\tau}\right)$ contains multiple candidate data $r_{\tau}$ centers, and $\operatorname{cen}\left(r_{\tau}\right)=u_{1}, u_{2}, \ldots, u_{\mathrm{n}}$ represents the set of candidate data centers that the data can be deployed. Because of the synchronization or data association between different data items in data sets, they need to transmit data frequently to form a relatively stable data traffic feature. The data transmission is deployed in the wireless transmission module designed above. Definition $F \rightarrow G$ is the mapping from data set $F$ to $G$. for any data $r_{\mathcal{E}}, r_{\varepsilon} \in F$, the mapping meets the following requirements:

$$
F \rightarrow G \mid\left(r_{i}\right) \in G \quad(1)
$$

size $\left(r_{\varepsilon}\right)<Q\left(u_{\mathrm{n}}\right)$ and $u_{\mathrm{n}} \in \operatorname{cen}\left(r_{\varepsilon}\right)$ are guaranteed to be met, and these two limits guarantee that data set $r_{\varepsilon}$ does not exceed the storage capacity limit of data center $u_{\mathrm{n}}$, and that it is placed within the range of its alternative data center. The specific data transmission deployment scheme is shown in Figure 1. 


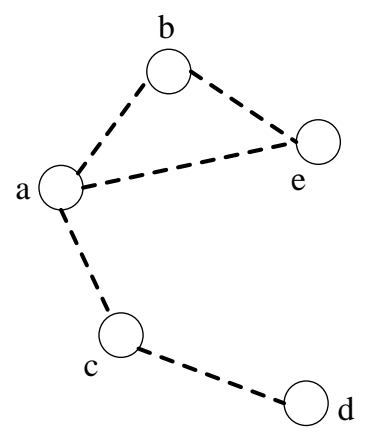

(a) Traffic between data sets

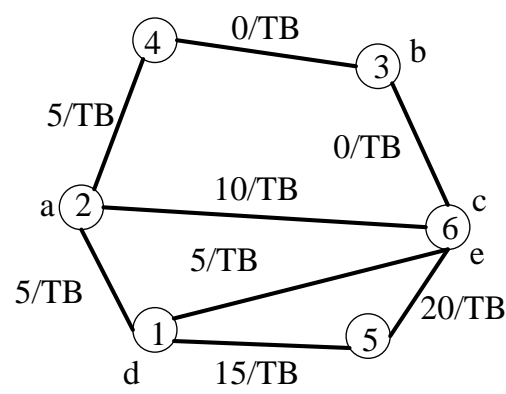

(b) Backbone network between data centers

Figure 1 Data transmission deployment

The data placement strategy in Figure 1 is $\left\{a \rightarrow u_{2}, b \rightarrow u_{3}, c \rightarrow u_{6}, d \rightarrow u_{1}, e \rightarrow u_{6},\right\}$. As long as the data center capacity limit is not exceeded, data $c$ and data $e$ can be deployed to the same data center $u_{6}$.

After the completion of data transmission deployment in enterprise information construction, the reliability analysis is carried out.

\section{Reliability analysis of enterprise information construction}

The reliability of enterprise information construction is analyzed from the path loss of data transmission channel. Path loss is defined as the difference between the effective transmit power and the received power, which represents the attenuation of the signal, and the unit is a positive value of $\mathrm{dB}$. Path loss consists of three parts: transmission loss, shadow fading, reflection loss and diffraction loss.

In practical work, such as deploying a data transmission channel, in order to ensure the reliability of the enterprise information process, the channel is generally calculated. One of the important contents is to calculate the transmission loss of the channel to measure the degree of path loss in the transmission process of data. With the increasing distance between the 
transmitting end and the receiving end, the intensity of electromagnetic wave will continue to decline. The path loss in free space refers to the attenuation degree of the transmitting electromagnetic wave when there is a completely unobstructed sight distance path between the receiving end and the transmitting end. The so-called free space refers to an infinite space full of even and lossless medium, which is isotropic, with conductivity of 0 and average relative permittivity and relative permeability of 1 . Assuming that the distance between the sender and the receiver in the free space is $\eta$, the input power of the data transmission process is $w_{1}$, the output power is ${ }^{w_{2}}$, and $\sigma$ represents the wavelength, the transmission loss is:

$$
Y_{s}=\frac{w_{1}}{w_{2}}=\left(\frac{4 \pi \eta}{\sigma}\right)^{2} \text { (2) }
$$

In the process of data transmission, the free space is a vacuum, and the transmission loss is the loss caused by the natural diffusion of energy with the increase of transmission distance.

The shadow effect is the loss caused by the influence of natural or human factors in the enterprise information construction. Shadow effect is easy to cause the level fading of sender and receiver, which is called shadow fading. The probability of shadow fading is as follows:

$$
L(\psi \geq x)=\frac{1}{c \sqrt{2 \pi}} \int_{0}^{\infty} 2 c^{2} d u
$$

In the formula, $\psi$ represents the decibel of the fading amount, and $c$ represents the standard deviation of the fading amount.

In the construction of enterprise information, reflection, diffraction and scattering will affect the transmission. In the process of data transmission, reflection occurs on the wall or obstacle surface. In the wireless channel, the influence of the ground reflection should also be considered. The ground reflection dual line model is shown in Figure 2 below.

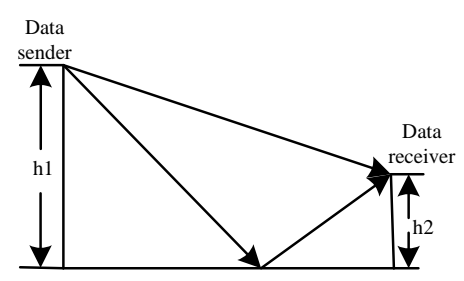

Figure 2 Two line model of ground reflection

From the two line model of ground reflection : 


$$
Y_{f}=w_{1} \frac{h_{1}^{2} h_{2}^{2}}{\eta}
$$

In the formula, $h_{1}$ represents the distance between the transmitting end and the ground, and $h_{2}$ represents the distance between the receiving end and the ground. $Y_{f}$ is the path loss during reflection.

Diffraction occurs when the moving path between the transmitter and the receiver is blocked by sharp edges. The secondary wave generated by the barrier surface spreads in the space, even around the back of the barrier. If the secondary wave bypasses the barrier and reaches the shadow area, this area is called "Fresnel area".

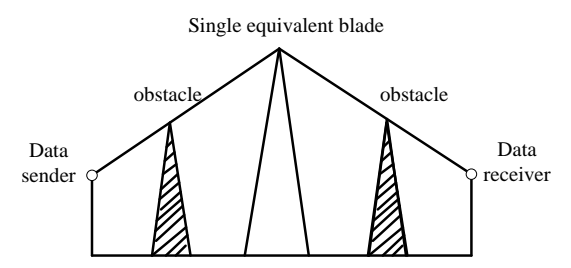

Figure 3 Equivalent single blade Burlington model

In wireless communication system, diffraction loss is caused by blocking secondary wave, that is, only a part of energy can bypass the blocking body. When there is more than one barrier in the transmission path, the diffraction loss caused by all the barriers must be calculated. In this way, the single edge diffraction model can be used to calculate the path loss.

$$
Y_{b}=\frac{1}{e \sqrt{2 \pi}} \exp \left(\frac{w_{1}}{\eta}\right)^{2}
$$

$w_{1}$ in the formula represents the path loss caused by diffraction.

The transmission loss, shadow fading probability and path loss in the process of reflection and diffraction are taken as the basis of reliability analysis. The standard range of path loss is determined according to the actual project size of enterprise informatization, so as to judge the reliability of enterprise informatization construction and complete reliability analysis.

\section{Design and analysis of simulation experiment}

\subsection{Experimental environment}

The hardware platform includes AT91 RM9200 development board, Siemens MC55 GPRS wireless communication module, and Linux host which is easy to develop and compile. While the method of building the software platform is embedded Linux as the experimental environment, the statistics of the experimental results are based on Windows system and the 
third party software.

The experiment mainly includes the packet loss in the process of data transmission. Before data transmission, basic settings must be completed. The client / server structure mode is adopted in the communication. The ARM board is used as the client PC as the Server, and the TCP protocol is used for data transmission. First, download the compiled program from the host computer to the development board, and set the corresponding execution authority. Set the IP in the Ping function to 211.129.11.102, the IP of the analog front-end computer to 211.86.39.54, and the port number to 80801 .

\subsection{Experiment setup}

The network topology between the experimental data centers is shown in Figure 4. After the unified simulation of the data deployment location, the unit data transmission cost of the link between data centers.

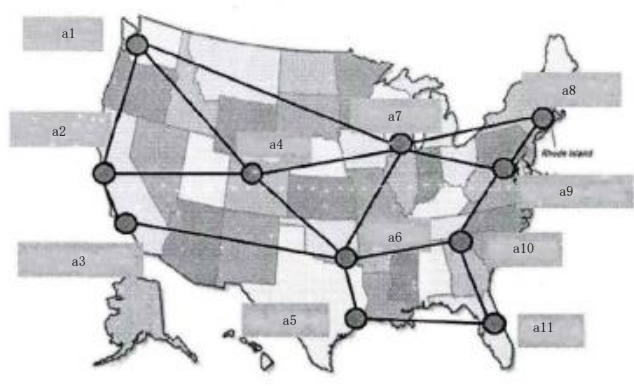

Figure 4 experimental network topology

As shown in Figure 4, it contains 11 data center nodes and 17 bidirectional links. For each bidirectional link, the free link capacity of the uplink and downlink is the same, the free bandwidth resource of each link is 10GB, and the transmission cost of each link is shown in Table 3. The free storage space of data center nodes is 100TB.

Table 3 Link data transmission cost between data centers

\begin{tabular}{llll}
\hline Link & Overhead & Link & Overhead \\
\hline$\left\{a_{1}, a_{2}\right\}$ & 30 & $\left\{a_{5}, a_{10}\right\}$ & 25 \\
$\left\{a_{1}, a_{4}\right\}$ & 3 & $\left\{a_{5}, a_{6}\right\}$ & 1 \\
$\left\{a_{1}, a_{7}\right\}$ & 25 & $\left\{a_{6}, a_{11}\right\}$ & 50 \\
$\left\{a_{2}, a_{4}\right\}$ & 6 & $\left\{a_{7}, a_{8}\right\}$ & 10 \\
$\left\{a_{2}, a_{3}\right\}$ & 15 & $\left\{a_{7}, a_{9}\right\}$ & 8 \\
$\left\{a_{3}, a_{6}\right\}$ & 35 & $\left\{a_{8}, a_{9}\right\}$ & 35 \\
$\left\{a_{4}, a_{7}\right\}$ & 15 & $\left\{a_{9}, a_{10}\right\}$ & 25 \\
\hline
\end{tabular}




\begin{tabular}{lll}
\hline $\left.\mathrm{a}_{4}, \mathrm{a}_{5}\right\}$ & 20 & $\left\{\mathrm{a}_{10}, \mathrm{a}_{11} 23\right.$ \\
& & \\
$\left\{\mathrm{a}_{5}, \mathrm{a}_{7}\right\}$ & 5 &
\end{tabular}

The scale of the data set used in the experiment increased from 10 to 100 , with a total of 10 groups. The size of each data set is randomly generated between 0.1 tb and 1TB, obeying the law of uniform distribution, and the traffic between data is randomly generated between $0.1 \mathrm{gb}$ and $1 \mathrm{~GB}$, obeying the law of uniform distribution.

\subsection{Experimental results and analysis}

In the above experimental environment and experimental settings, the experimental results of different methods are shown in Figure 5.

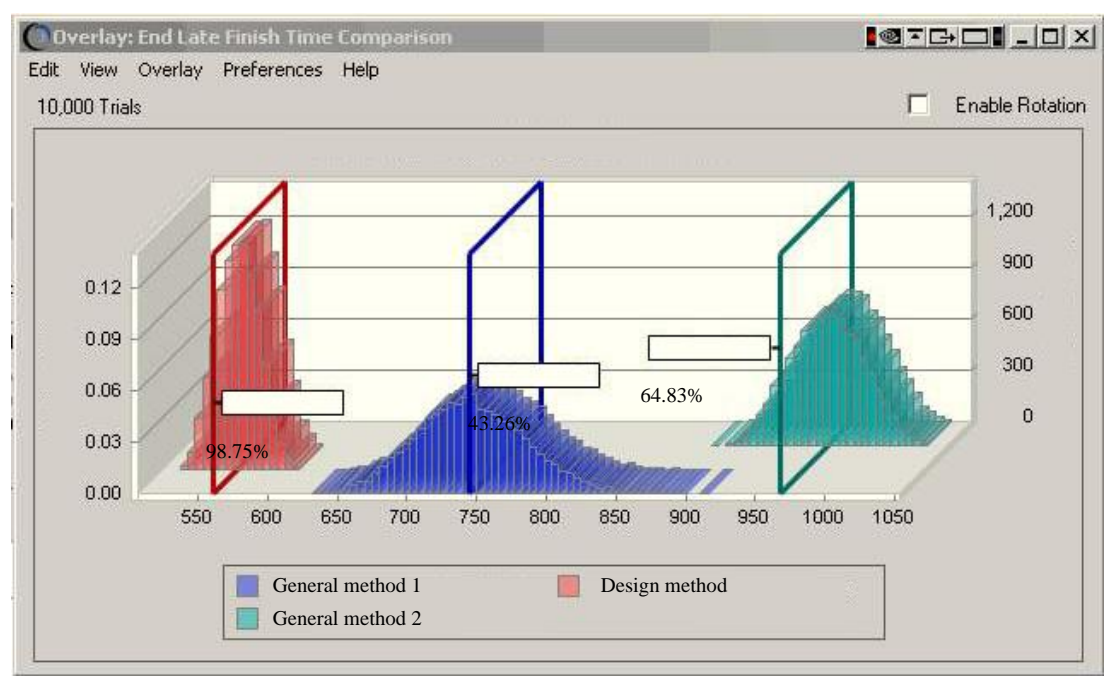

Figure 5 experimental comparison results of different methods

Observing the results in Figure 5, it is shown that data transmission deployment and reliability analysis are carried out by using conventional methods 1 and 2, and packet loss rate is low; while data transmission deployment and reliability are carried out by using the designed method, no packet loss occurs, and the packet loss rate is $0 \%$. Compared with different methods, the design of data transmission deployment and reliability analysis method is better than the conventional method.

\section{Conclusion}

In the enterprise information construction, data transmission deployment is an important part. Its good or bad deployment directly affects the completion of enterprise information and the subsequent development of the enterprise. It is of great significance for the development of the enterprise to use certain means to analyze the reliability of enterprise information 
construction and strictly control the quality of enterprise information construction. In view of the problems existing in the traditional methods, after the completion of the method design in this paper, a comparative experiment is designed to prove that the design method solves the problems existing in the traditional methods and provides some help for the enterprise information construction.

In the future, the wireless network will deploy more than 10 times more wireless nodes than the existing stations. Within the coverage area of the macro station, the distance between stations will be kept within $10 \mathrm{~m}$, and support 25000 users in every $1 \mathrm{~km} 2$. At the same time, the ratio of the number of active users and the number of stations may reach 1:1, that is, users and service nodes correspond one by one. The densely deployed network shortens the distance between terminals and nodes, greatly improves the power and spectrum efficiency of the network, expands the network coverage, expands the system capacity, and enhances the flexibility of services in different access technologies and coverage levels. Although the super dense heterogeneous network architecture has a great development prospect in $5 \mathrm{~g}$, the reduction of the distance between nodes, the more dense network deployment will make the network topology more complex, which can be further studied in the future.

\section{Reference}

[1] QIN Weirong. Construction of informationalized Internet of things under cloud computing and big data [J]. Laser Journal, 2018,39(05):120-123.

[2] ZHANG Xintong, SHI Xiaoyang, SHI lei,et al. Discussion on the development of blood platform informationization construction in Shandong Province [J]. Chinese Journal of Blood Transfusion, 2018,31(09):1073-1076.

[3] XU Xiaoqi, LI Yanling . Synergies of informatization and agricultural modernization in China from the provincial perspective and the data from 2000 to 2016 [J]. Journal of Hunan Agricultural University(Social Sciences), 2019,20(03):58-66.

[4] MA Zhaoting, LIU Yong, SHEN Jianming,et al. Thinking of construction issue on spatio-temporal big data and platform for smart city [J]. Science of Surveying and Mapping, 2019,44(06):279-284

[5] TIAN Hao, ZHAO Xiaohu, ZHANG Kai,et al. Design of data transmission system in coal mine underground based on 6LowPAN [J]. Industry and Mine Automation, 2019,45(08):6-12.

[6] LYU Na, LIU Chuang, CHEN Kefan,et al. A method for centralized control network deployment of aeronautic swarm [J]. Acta Aeronautica ET Astronautica Sinica, 2018,39(07):172-184.

[7] YUAN Hui, ZHA Zhiyong. Optimized Monitoring Network Measurement Point Data Transmission Efficiency Mathematical Modeling Simulation [J]. Computer Simulation, 2019,36(05):218-221.

[8] WANG Wei . A Low Energy Consumption Cloud Computing Virtual Machine Deployment Scheme

[J]. Control Engineering of China, 2018,25(07):1291-1296. 
[9] LI Xingyun, QI Jinping . Reliability analysis of multi pantograph system based on fuzzy Bayesian network [J]. Journal of Railway Science and Engineering, 2018,15(06):1383-1390.

[10] Yao Li, Zhang Jiangming, Hu Yingjun,et al. Study on Running Reliability Data Processing Method for Smart Electrical Energy Meters [J]. Bulletin of Science and Technology, 2018,34(10):125-129. 